Algorithmische Prognose und ihre Anwendung in der Justiz An den Beispielen der COMPAS-Software und im Bereich der Entschädigung für Personenschäden

WU Yiyue

Vor dem Hintergrund der raschen Entwicklung von Internet, Big Data und künstlicher Intelligenz und ihrer Interaktion hinterlässt jede menschliche Bewegung elektronische Spuren, nämlich Daten, die verarbeitet, analysiert und genutzt werden können. ${ }^{1}$ Durch diese Daten ist es möglich, das körperliche Verhalten und sogar innere Aktivitäten einer Person in der Vergangenheit bis zu einem gewissen Grad zu kennen. Zum Beispiel können die Aktivitäten einer Person durch GPS-Daten des Mobiltelefons, Dashcam-Aufnahmen, Überwachungsvideos usw. wiederhergestellt werden und durch Webbrowsing-Aufzeichnungen, Suchinformationen in Suchmaschinen, Browsing- und Einkaufsaufzeichnungen von Online-Handelsunternehmen usw. kann man Bedenken und persönliche Vorlieben einer Person erkennen. Es ist keine Übertreibung zu sagen, dass alles auf der Welt, einschließlich des Menschen, in den Augen von Algorithmen eine Sammlung von Daten ist. ${ }^{2}$ Wir sind bereits in eine Ära eingetreten in der alles kalkuliert werden kann. ${ }^{3}$ Die Begegnung von künstlicher Intelligenz, Big Data und Recht bringt einerseits Risiken und Herausforderungen für die traditionelle menschliche Gesellschaft mit sich und erfordert Recht, um sie zu regulieren. Andererseits schafft sie auch Impulse für die Gesetzgebung, Justiz und Verwaltung und fördert sie, um beispiellos große Veränderungen zu erreichen. ${ }^{4}$

1 ZHENG Ge (郑戈), Das Recht der Algorithmen und der Algorithmus des Rechts (算法的法律与法律的算法), China Law Review (中国法律评论) 2018, Nr. 2, S. 68 .

2 John Cheney-Lippold, We Are Data: Algorithms and the Making of Our Digital Selves, New York 2017, S. 251.

3 MA Changshan (马长山), Soziale Risiken der künstlichen Intelligenz und ihre rechtliche Regulierung (人工智能的社会风险及其法律规制), Science of Law (法 律科学) 2018, Nr. 6, S. 49.

4 WANG Qinghua (汪庆华), Der Weg zur rechtlichen Regulierung der künstlichen Intelligenz: Eine Rahmendiskussion (人工智能的法律规制路径：一个框架性讨 论), Modern Law Science (现代法学) 2019, Nr. 2, S. 54. 


\section{Entscheidungshilfe und algorithmische Entscheidung}

Die Rechtsklarheit und die Vorhersehbarkeit von Verhaltensfolgen sind die Grundmerkmale eines Rechtsstaats. ${ }^{5}$ Auf der Grundlage der wissenschaftlichen Analyse der gesetzlichen Bestimmungen und des Zusammenhangs zwischen zahlreichen vorherigen Fällen und rechtlichen Konsequenzen sollte es daher möglich und vertrauenswürdig sein, die rechtlichen Konsequenzen anhängiger Fälle vorherzusagen. Momentan ist es bereits möglich, Rechtsinformationen durch künstliche Intelligenz und Big Data eingehend zu analysieren und auf dieser Grundlage Vorhersagen über rechtliche Konsequenzen zu treffen. ${ }^{6}$ Daher kann die künstliche Intelligenz die Arbeit des Gerichts erheblich entlasten und die Gerichtsentscheidungen unterstützen.

\section{Entscheidungshilfe durch künstliche Intelligenz und ibre Probleme}

Zurzeit konzentriert sich die Anwendung der Entscheidungshilfesysteme in der VR China hauptsächlich auf den Bereich der Strafbemessung. Viele Gerichte haben solche Systeme bereits in der Praxis angewendet. ${ }^{7}$ Die Systeme extrahieren und strukturieren automatisch die Fallinformationen in den Rechtsdokumenten, verwenden mathematische Modelle zur Beschreibung der Merkmale der Daten und verwenden Algorithmen zur Berechnung verschiedener Variablen, um die Strafbemessungsergebnisse abzuleiten. ${ }^{8}$ Dies wird zweifellos dazu beitragen, repetitive Arbeit zu reduzieren und die Arbeitseffizienz zu erhöhen. Doch ihre zunehmende Verwendung in der Praxis hat auch Besorgnis bei Wissenschaftlern erregt. Obwohl solche Systeme mit Wörtern wie „Hilfe“, „Empfehlung“ oder „Vorhersage“

5 BAI Jianjun (白建军), Studie zur Vorhersage von Strafbemessung auf der Grundlage der kollektiven Erfahrung von Richtern (基于法官集体经验的量刑预测研究), Chinese Journal of Law (法学研究) 2016, Nr. 6, S. 140.

6 BAI Jianjun (白建军), Möglichkeit und Grenzen der Vorhersage von Entscheidungen im Zeitalter legaler Big Data (法律大数据时代裁判预测的可能与限度), Exploration and Free Views (探索与争鸣) 2017, Nr. 10, S. 95 f.

7 Vgl. ZHAO Hui (赵慧), Hubei: Visualisierung des Strafbemessungsprozesses (湖 北：量刑建议过程可视化), Security Daily (检察日报) 22.08.2020, S. 3; ZHANG Lingfeng (张凌锋), Nansha: Intelligenter Vergleich und präziser Push (南沙 : 智能 比对精准推送), Security Daily (检察日报) 22.08.2020, S. 3.

8 WANG Lusheng (王禄生), Technische Hindernisse für justizielle Big Data und die Entwicklung künstlicher Intelligenz (司法大数据与人工智能开发的技术障碍), China Law Review (中国法律评论) 2018, Nr. 2, S. 50. 
bezeichnet werden, ist es nicht zu leugnen, dass sie tatsächlich selbstständig Entscheidungen generieren können. In diesem Fall muss man darüber nachdenken, ob es sich um einen menschlichen Richter oder einen Algorithmus handelt, der die Justizhoheit ausübt. Momentan ist es in China, Deutschland, den USA usw. nicht erlaubt, dass die künstliche Intelligenz als Richter fungiert. Zum Beispiel zielen das Richtergesetz der VR China, ${ }^{9}$ das Deutsche Richtergesetz und der Code of Conduct for United States Judges in den USA offensichtlich nur auf menschliche Richter ab, nicht auf Roboter mit künstlicher Intelligenz.

Wenn der Richter bei der Vorbereitung einer Entscheidung nur Hilfsmittel wie intelligente Datenbanken verwendet, ist davon auszugehen, dass die Entscheidung von dem Richter in unabhängiger Weise getroffen wird. Wenn der Richter dagegen den gesamten Vorbereitungsprozess der künstlichen Intelligenz überlassen und nur die Ergebnisse akzeptieren und verkünden würde, dann wäre es zweifellos sehr problematisch. Die Grenze zwischen diesen beiden Situationen ist jedoch in der Praxis häufig unklar und schwer zu unterscheiden. Insbesondere, wenn das Vertrauen von Menschen in künstliche Intelligenz wächst, und unter dem Einfluss von Mechanismen wie einer „Abweichungswarnung“ auf die unabhängige Entscheidung von Richtern, werden Richter wahrscheinlich auch keine Entscheidung treffen, die sich vom Ergebnis von Entscheidungshilfesystemen unterscheidet. ${ }^{10}$ In Frankreich gab es Fälle in denen Anwälte Berufung einlegten, weil sich die Entscheidung des erstinstanzlichen Gerichts von der simulierten Entscheidung durch künstliche Intelligenz und Big Data unterschied. Sie forderten die ursprüngliche Entscheidung aufzuheben, um eher mit dem Entscheidungshilfesystem übereinzustimmen. ${ }^{11}$ Es gibt zahlreiche Anzeichen dafür, dass solche Entscheidungshilfesysteme wahrscheinlich allmählich die Justizhoheit untergraben. Wenn das unabhängige Gerichtsverfahren zu einer bloßen Formalität würde, dann wären diese

9 中华人民共和国法官法 v. 23.4.2019, Amtsblatt des ständigen Ausschusses des Nationalen Volkskongresses（全国人民代表大会常务委员会公报）2019，Nr.3， S. 522 ff.; deutsche Übersetzung siehe Anne Sophie Ortmanns (Übersetzerin), in: Zeitschrift für Chinesisches Recht 2019, S. 405 ff.

10 Peter Enders, Einsatz künstlicher Intelligenz bei juristischer Entscheidungsfindung, in: Juristische Arbeitsblätter 2018, S. 723.

11 SHI Pengpeng (施鹏鹏), Können Entscheidungen durch Big Data vorhergesagt werden? (裁判可通过大数据预测? ), Security Daily (检察日报) 30.1.2018, S.3. 
algorithmischen Systeme tatsächlich zu „Alpha-Richtern“12 geworden, die die menschliche Justizhoheit ausüben.

\section{Vergleich zwischen algorithmischer Entscheidung und Entscheidung des Richters}

Obgleich die algorithmische Entscheidung bis jetzt noch verboten ist, ist dies nicht die Grenze des wissenschaftlichen Denkens. Im Gegenteil ist es notwendig, das Verständnis und die Erkenntnis über die algorithmische Entscheidung zu verstärken und sich im Voraus auf die grundlegenden Änderungen vorzubereiten, denen das Recht in Zukunft begegnen könnte. Die Juristen müssen zuerst darüber nachdenken, welche Vor- und Nachteile die algorithmische Entscheidung gegenüber der Entscheidung des Richters hat.

Erstens ist die algorithmische Entscheidung stabiler und vorhersehbarer. ${ }^{13}$ Denn wenn ein Algorithmus eine Entscheidung zu treffen hat, berücksichtigt er nur die Kriterien, die nach dem Programmdesign zulässig sind, und wandelt die Fakten im Fall für genaue Berechnungen in mathematische Variablen um. Mit anderen Worten steht dem Algorithmus kein Ermessensspielraum zu. Obwohl sich der Richter bei seiner Entscheidung auch an viele festgelegte Standards halten muss, kann der Richter bewusst oder unbewusst von anderen Faktoren beeinflusst werden. Daher ist die algorithmische Entscheidung förderlicher für die Verwirklichung des Mottos: „ähnliche Fälle werden ähnlich entschieden“.

Zweitens wird die Einzigartigkeit des Individuums bei der algorithmischen Entscheidung ignoriert und durch die gemeinsamen Merkmale der Gruppe ersetzt. ${ }^{14}$ Die Berechnungen der Algorithmen basieren auf verschiedenen Variablen, die durch Big Data erhalten werden und deren Wechselwirkungen. ${ }^{15}$ Wenn der Algorithmus in einem Fall mit einer Partei konfrontiert wird, sucht er in der Datenbank nach Daten, die

12 HE Fan (何帆), Wie weit sind wir von „Alpha-Richtern“ entfernt? (我们离“阿尔 法法官”还有多远?), Zhejiang People’s Congress Magazine (浙江人大) 2017, Nr. 5, S. 47.

13 Christian Ernst, Algorithmische Entscheidungsfindung und personenbezogene Daten, in: JuristenZeitung 2017, S. 1027.

14 Klaus Lenk, Die neuen Instrumente der weltweiten digitalen Governance, in: Verwaltung und Management 2016, S. 233.

15 Phillip Richter, Big Data, Statistik und die Datenschutz-Grundverordnung, in: Datenschutz und Datensicherheit 2016, S. 581. 
mit allen Aspekten der Informationen der Partei übereinstimmen, und verwendet dann deduktive Methoden zur Entscheidung. Daher kann der Algorithmus eine bestimmte Partei nicht als einziges Individuum betrachten, sondern nur als Mitglied einer Gruppe, zu der sie gehört. Ob dies den Anforderungen der Gerechtigkeit entspricht, ist sehr fraglich. ${ }^{16}$ Die Entscheidung des Richters basiert dagegen auf der Interpretation des Falls durch den Richter und kann flexibel an die verschiedenen Situationen im Fall angepasst werden. ${ }^{17}$ In dieser Hinsicht entspricht die Entscheidung des Richters eher den wesentlichen Merkmalen der Rechtsverfahren und fördert die Verwirklichung der Gerechtigkeit in Einzelfällen.

Drittens fehlt dem Algorithmus die Fähigkeit zur Selbstreflexion und er kann die aktuelle Situation der Gesellschaft nur widerspiegeln. Im Jahr 2016 hat Kabir Alli aus Virginia, USA, ein kurzes Video in den sozialen Medien hochgeladen, das in der gesamten Gesellschaft große Besorgnis und Diskussion hervorrief: Wenn man "three black teenagers" in die Google-Suche eingab, dann wurden meistens die sogenannten Mugshots angezeigt, also die Gesichtsfotos von schwarzen Jugendlichen, die die Polizei archiviert hat, um Kriminelle zu identifizieren. Aber wenn man „three white teenagers" eingab, wurden Fotos von weißen Jugendlichen mit fröhlichem Lächeln angezeigt, die von professionellen Fotografen aufgenommen wurden. ${ }^{18}$ Daher wurde Google von vielen Menschen als rassistisch kritisiert. Die späteren Untersuchungsergebnisse zeigten jedoch, dass der Grund für die oben genannte Situation nicht das Programmdesign der Google-Suche war, sondern unsere soziale Realität: die Fotos von lächelnden weißen Jugendlichen wurden häufig für Werbezwecke verwendet, weshalb sie auf Nachrichtenseiten, in sozialen Medien und in anderen wichtigen Big-Data-Quellen weit verbreitet waren. Aber die Lebensfotos von schwarzen Teenagern wurden ziemlich selten für Werbung verwendet, sodass Suchmaschinen archivierte Fotos der Polizei leichter finden konnten. Daher wurde die bestehende oder sogar unbewusste Diskriminierung zwischen Schwarzen und Weißen in der Gesellschaft durch Big Data aufgedeckt und angezeigt, was zu scheinbar rassistischen Suchergebnissen

16 Hermann Hill, Scientific Regulation - Automatische Verhaltenssteuerung durch Daten und Algorithmen, in: Hermann Hill/Utz Schliesky (Hrsg.), Auf dem Weg zum digitalen Staat - auch ein besserer Staat?, Baden-Baden 2015, S. 274.

17 Christian Ernst (Fn. 13), S. 1028; JI Weidong (季卫东), Dialektische Ansichten zur Strafbemessung von Computern (电脑量刑辩证观), Tribune of Political Science and Law (政法论坛) 2007, Nr. 1, S. 126.

$18<$ https://www.zeit.de/digital/internet/2016-06/google-three-black-teenagers-suchm aschine-rassismus $>$ eingesehen am 3.7.2021. 
führte. Dies zeigt, dass der Algorithmus nur die Informationen darstellen und verarbeiten kann, die er im Internet erhält. Er kann aber nicht prüfen, ob das Ergebnis dem Recht, der Ethik und der Moral entspricht. Wenn diese Konstellation weiterhin besteht oder sich sogar verschlechtert, wird dieser Eindruck im Algorithmus fixiert, was schließlich zu diskriminierenden Entscheidungsergebnissen führen kann. Daher besteht die grundlegende Lösung für dieses Problem nicht darin, den Algorithmus zu ändern, sondern die soziale Situation zu verbessern.

Zusammenfassend lässt sich sagen, dass die algorithmische Entscheidung gegenüber der Entscheidung des Richters einige Vorteile hat, aber auch große Nachteile aufweist. Sie entspricht den Anforderungen von Gerichtsverfahren nicht vollständig und ist momentan auf rechtlicher Ebene nicht zulässig. Obwohl das Entscheidungshilfesystem, beispielsweise das Strafbemessungssystem, ein intelligentes Instrument darstellt, das vom Richter gesteuert wird, hat es in der Praxis möglicherweise die Entscheidung des Richters bis zu einem gewissen Grad ersetzt. Dies erfordert auf jeden Fall Wachsamkeit!

\section{Algorithmische Prognose und ibr justizieller Wert}

\section{Die Prognosefunktion des Algorithmus}

Mit der Kombination von Big-Data-Analyse und Algorithmen können künstliche Intelligenzsysteme möglicherweise zukünftige Ereignisse (einschließlich menschlichen Verhaltens) in sehr kurzer Zeit vorhersagen und die Genauigkeit ist viel höher als bei der herkömmlichen manuellen Vorhersage. ${ }^{19}$ Manche Wissenschaftler glauben, dass der transformative Wert von Big Data und Analytics in ihren Vorhersagekapazitäten liege. ${ }^{20}$ Zum Beispiel im industriellen Bereich können durch das Sammeln von „Industrie-Daten“ wie Arbeitsstunden einer Maschine, Druck, Temperatur, Kraftstoffdurchfluss, Stromverbrauch und ungeplanten Ausfallzeiten, in Verbindung mit den produzierten Produktionsgütern, Tag- und Nachtschichten, der Auslastung, dem Wetter usw. die Abnutzung von Verschleißteilen

19 Hervais Simo, Big Data: Opportunities and Privacy Challenges, in: Philipp Richter (Hrsg.), Privatheit, Öffentlichkeit und demokratische Willensbildung in Zeiten von Big Data, Baden-Baden 2015, S. $28 \mathrm{f}$.

20 Jennifer Bachner, Predictive Policing: Preventing Crime with Data and Analytics, Washington D.C. 2013, S. 14. 
und deren erwartete Lebensdauer angezeigt werden, um den Ausfall der Maschine genau vorherzusagen (auf den Tag genau bis zu drei Monaten im Voraus). ${ }^{21}$ Dies hilft Maschinenmanagern, Maßnahmen im Voraus zu treffen und Verluste durch plötzliche Ausfälle zu vermeiden.

Big Data und Algorithmen können nicht nur vorhersagen, wann eine Maschine ausfällt, sondern auch „Ausfälle“ in der menschlichen Gesellschaft vorhersagen, worauf das sog. Predictive Policing in den USA basiert. Im Jahr 2011 verwendeten Forscher der University of California, Los Angeles und der Santa Clara University den Algorithmus zur Vorhersage der Nachbeben von Erdbeben als Basis und speisten die Daten von 13 Millionen Delikten aus den vergangenen acht Jahrzehnten in das zugrundeliegende mathematische Modell ein. Der Datenbestand wird ständig durch neue, aktuelle Straftaten ergänzt. ${ }^{22}$ Das Programm kann 15 Quadratflächen mit einer Seitenlänge von ca. 150 Metern mit der höchsten Wahrscheinlichkeit von Straftaten auf der Landkarte abgrenzen, ${ }^{23}$ sodass die Polizei im Voraus vorbeugende Maßnahmen ergreifen und Gegenmaßnahmen in diesen Bereichen vorbereiten kann. Es ist ersichtlich, dass die algorithmische Prognose unvergleichliche Vorteile hinsichtlich der Breite und Genauigkeit hat und eine sehr wichtige Rolle bei der sozialen Steuerung spielen kann.

\section{Risikobewertungssoftware in amerikanischen Strafverfahren}

Der Richter muss bei der gerichtlichen Entscheidung auch manchmal Vorhersagen erzielen. Wenn das Algorithmus-System zu diesem Zeitpunkt genauere und effizientere Vorhersagen treffen kann, wird es zweifellos die richtige Hilfskraft des Richters. In den letzten Jahren hat mehr als die Hälfte der US-Bundesstaaten begonnen, Risikobewertungssoftware wie COMPAS, PSA und LSI-R zu verwenden, um Richter bei der Strafbemes-

21 Christopher Peschel/Sebastian Rockstroh, Big Data in der Industrie: Chancen und Risiken neuer datenbasierter Dienste, in: Multimedia und Recht 2014, S. 571.

22 Martin Steinebach/Christian Winter/Oren Halvani/Marcel Schäfer/York Yannikos, Big Data und Privatheit, in: Michael Waidner (Hrsg.), Begleitpapier Bürgerdialog: Chancen durch Big Data und die Frage des Privatsphärenschutzes, Stuttgart 2015, S. $13 \mathrm{f}$.

23 Jennifer Bachner (Fn. 20), S. 25. 
sung zu unterstützen. ${ }^{24}$ Sie unterscheiden sich von den oben erwähnten Entscheidungshilfesystemen, da diese Risikobewertungssoftware die Strafe nicht direkt empfiehlt, sondern nur die Rückfallwahrscheinlichkeit vorhersagt und somit eine wichtige Referenz für den Richter anbietet.

Solche Risikobewertungssoftwares werden in der Praxis ebenfalls stark angezweifelt. „State v. Loomis“ ist ein typischer Fall dafür. Eric Loomis wurde strafrechtlich verfolgt, weil er illegal auf ein Auto schoss. Während der Vorbereitung der Strafbemessung erstellte ein Beamter der Abteilung für Korrekturen in Wisconsin einen Untersuchungsbericht, der die Ergebnisse der Risikobewertung von Loomis durch die COMPAS-Software enthielt. Diese Einschätzung basierte auf einem Interview mit Loomis und seiner früheren Kriminalgeschichte. Das Gericht bezog sich bei der Bekanntgabe des Urteils auf den COMPAS-Bewertungsbericht und verurteilte Loomis auf der Grundlage dieses Berichts zu sechs Jahren Haft und fünf Jahren erweiterter Aufsicht. ${ }^{25}$ Danach reichte Loomis einen Antrag auf Erleichterung ein und machte geltend, dass das Vertrauen des Gerichts in COMPAS seine Prozessrechte verletzt habe. ${ }^{26}$ Weil COMPAS-Berichte nur Daten liefern, die nur für bestimmte Gruppen relevant sind und weil die zur Erstellung der Berichte verwendete Methodik ein Geschäftsgeheimnis ist, machte Loomis geltend, dass die Verwendung der COMPAS-Bewertung durch das Gericht sowohl sein Recht auf eine individuelle Strafe als auch sein Recht aufgrund genauer Informationen verurteilt zu werden verletze. ${ }^{27}$ Aber Richterin Ann Walsh Bradley wies die Argumente von Loomis wegen des ordnungsgemäßen Verfahrens zurück ${ }^{28}$ und erklärte, dass die Verurteilung, die eine COMPAS-Bewertung berücksichtigt, immer noch ausreichend individualisiert sei, da der Bericht nicht die einzige Grundlage für eine Entscheidung sei und die Gerichte über das Ermessen und die Informationen verfügen, die erforderlich sind, um der Bewertung gegebenenfalls nicht zuzustimmen. ${ }^{29}$

Dieser Fall hat weltweit viele Diskussionen ausgelöst und einer der Schwerpunkte ist die Opazität solcher Risikobewertungssoftware. Da der Algorithmus hinter der COMPAS-Software ein Geschäftsgeheimnis ist,

24 Benjamin L. Liebman (李本), Künstliche Intelligenz in der amerikanischen Justizpraxis: Probleme und Herausforderungen (美国司法实践中的人工智能：问题与 挑战), China Law Review (中国法律评论) 2018, Nr. 2, S. 54.

25 State v. Loomis, N. W. 2d at 755-756.

26 State v. Loomis, N. W. 2d at 756.

27 State v. Loomis, N. W. 2 d at 757.

28 State v. Loomis, N. W. 2 d at 757.

29 State v. Loomis, N. W. 2d at 764-765. 
werden das Gericht und der Angeklagte nur über das Bewertungsergebnis, also die vorhergesagte Rückfallwahrscheinlichkeit, informiert, nicht jedoch über den Bewertungsprozess. Dass der Algorithmus nicht offen ist, ist nur ein Zeichen der Opazität. Tatsächlich liegt die tiefere Opazität in der sogenannten „Undurchdringbarkeit des maschinellen Lernens“, die ein inhärentes technisches Merkmal des Algorithmus-Systems ist. ${ }^{30}$ Mit anderen Worten, selbst wenn der Quellcode eines Algorithmus-Systems veröffentlicht wird, bedeutet dies nicht, dass Menschen den Code lesen und verstehen können. ${ }^{31}$ Es gibt zwei Hauptgründe für diese Situation: Erstens ist der Code oft extrem kompliziert und leicht verwirrend. Selbst wenn Experten ihn analysieren, übersehen sie oft viele Probleme. Beispielsweise war die in 2014 der Öffentlichkeit bekanntgewordene Sicherheitslücke „Heartbleed“ eine der schwerwiegendsten Informationssicherheitslücken in der Geschichte und für die meisten Internetnutzer eine potenziell katastrophale Sicherheitslücke. Sie wurde durch einen häufigen Programmierfehler verursacht, obwohl der Fehler den Open-Source-Überprüfungsprozess bestand und zwei Jahre lang existierte und es jedem (einschließlich technischer Experten) in diesem Zeitraum möglich war, den Code zu lesen und zu analysieren. ${ }^{32}$ Zweitens werden bei der statischen Analyse von reinem Quellcode in der Regel keine Pfadabhängigkeiten überprüft, das heißt, andere Softwares bleiben unklar, die für den korrekten Betrieb des zu überprüfenden Programms erforderlich sind, sodass es leicht zu unvollständigen oder falschen Schlussfolgerungen kommen kann. Denn wenn sich dieselbe Codezeile mit verschiedenen externen Systemen verbindet, hat sie wahrscheinlich völlig unterschiedliche Bedeutungen, was zu völlig unterschiedlichen Effekten führt. ${ }^{33}$ Daher ist unbekannt, wie diese Algorithmus-Systeme die Ergebnisse erhalten, sodass Zweifel aufgrund ihrer Opazität immer da sein werden.

Aus diesen Gründen werden diese algorithmischen Systeme als „Black Box" bezeichnet: Man kann nur die Ein- und Ausgabe des Systems beob-

30 Mario Martini, Blackbox Algorithmus - Grundfragen einer Regulierung Künstlicher Intelligenz, Berlin 2019, S. 41.

31 L. Jean Camp, Varieties of Software and Their Implications for Effective Democratic Government, in: Proceedings of the British Academy 2006, Bd. 135, S. $183 \mathrm{ff}$.

32 Joshua A. Kroll/ Joanna Huey/Solon Barocas/Edward W. Felten/Joel R. Reidenberg/David G. Robinson/Harlan Yu, Accountable Algorithms, in: University of Pennsylvania Law Review 2017, Bd. 165, S. 647.

33 Mario Martini (Fn. 30), S. 41; Joshua A. Kroll/ Joanna Huey/Solon Barocas/ Edward W. Felten/Joel R. Reidenberg/David G. Robinson/Harlan Yu (Fn. 32), S. 648. 
achten, aber der tatsächliche Betriebsprozess dazwischen ist unbekannt. ${ }^{34}$ Eine Untersuchung ergab, dass die in den USA verwendete Software zur Vorhersage des Rückfallrisikos möglicherweise zu Rassendiskriminierung neigt: Im Vergleich zu Weißen ist das vom System geschätzte Rückfallrisiko von Schwarzen erheblich höher als das tatsächliche Rückfallrisiko. Der Unterschied zwischen der vom System vorhergesagten Rückfallrate und der tatsächlichen Rückfallrate bei Schwarzen ist doppelt so hoch wie bei Weißen. ${ }^{35}$ Damit wird die Besorgnis über die Opazität des Algorithmus und die Diskriminierung von Algorithmen noch lange bestehen, obwohl es effizient ist, das Rückfallrisiko durch Algorithmen vorherzusagen.

\section{Die Perspektive der Anwendung algorithmischer Prognose im Bereich der Entschädigung für Personenschäden}

Die Gerichte in China haben bereits bei manchen Zivil- und Handelssachen einige intelligente Instrumente verwendet. Beispielsweise können die intelligenten Systeme in bestimmten Schadenersatzfällen bereits automatisch die Fallinformationen erkennen, kombiniert mit der notwendigen manuellen Kontrolle kann die endgültige Entschädigungssumme automatisch berechnet werden. ${ }^{36} \mathrm{Da}$ die relevanten Gesetze oder justiziellen Auslegungen eine klare Methode zur Berechnung der Entschädigungshöhe vorschreibt, kann diese durch Datenidentifikation in eine technische Aufgabe umgewandelt werden, um abweichungsarme Schlussfolgerungsergebnisse zu erzielen. ${ }^{37}$ Dadurch können Auslassungen und Fehler effektiv vermieden und Arbeits- und Zeitkosten für Fälle mit mehr Ausgleichsposten eingespart werden. Die Funktion von Algorithmen der künstlichen Intelligenz im Bereich des Schadensersatzes sollte jedoch nicht bis hier aufhören und auch die für Richter schwierig zu bewältigende Prognoseaufgabe übernehmen. Da das Zivil- und Handelsrecht nicht in derart direktem

34 Frank Pasquale, The Black Box Society: The Secret Algorithms That Control Money and Information, Cambridge, MA/London 2015, S. 3.

$35<$ https://www.propublica.org/article/machine-bias-risk-assessments-in-criminal-sen tencing $>$ eingesehen am 5.7.2021.

36 TAN Shigui (谭世贵)/WANG Qiang (王强), Die Praxis, Probleme und Gegenmaßnahmen des Aufbaus von Smart Courts in China (我国智慧法院建设的实 践、问题与对策), Journal of Hangzhou Normal University (Social Science Edition) (杭州师范大学学报<社会科学版>) 2019, Nr. 6, S. 111.

37 LONG Fei (龙飞), Anwendung und Entwicklung von künstlicher Intelligenz im Bereich der Streitbeilegung (人工智能在纠纷解决领域的应用与发展), Science of Law (法律科学) 2019, Nr. 1, S. 58. 
Zusammenhang mit dem menschlichen Leben, der Freiheit, den nationalen Zielen und der sozialen Gerechtigkeit steht, wie das Strafverfahren, ${ }^{38}$ lassen sich dort möglicherweise besser Prognoseaufgaben übernehmen.

Im Bereich der Entschädigung für Personenschäden ist die Berechnung des Todes- bzw. Behinderungsentschädigungsgeldes eine große Schwierigkeit. Die grundlegende Idee dafür besteht darin, die durch den Unfalltod oder die Behinderung des Opfers seinen nahen Verwandten verursachten Vermögensschäden zu berechnen. ${ }^{39}$ Mit anderen Worten, es wird ermittelt, wie viel Einkommen in Zukunft erzielt hätte werden können, wenn das Opfer nicht verstorben oder behindert geworden wäre.

$\mathrm{Da}$ das Stadt-Land-Doppelmodell ${ }^{40}$ in der vom Obersten Volksgericht 2003 verkündeten „Auslegung des Obersten Volksgerichts zu einigen Fragen der Rechtsanwendung bei der Behandlung der Entschädigung für Personenschäden" 41 weit verbreitete Zweifel und Unzufriedenheit mit dem sog. „Unterschiedlichen-Preis-für-gleiches-Leben-Problem“ hervorgerufen hat, verwandelt sich das System zur Entschädigung für Personenschäden in China derzeit zu einem einheitlichen Modell, das sich auf die Verwendung einheitlicher Standards für die Berechnung des Todes- und Behinderungsentschädigungsgeldes für Stadt- und Landbewohner bezieht. Im April 2019 wurde die „Stellungnahme des Zentralkomitees der Kommunistischen Partei Chinas und des Staatsrates zur Einrichtung und Vervoll-

38 JI Weidong (季卫东), Die Veränderung der Justizhoheit im Zeitalter der künstlichen Intelligenz (人工智能时代的司法权之变), Oriental Law (东方法学) 2018, Nr. 1, S. 128.

39 HUANG Songyou (黄松友) (Hrsg.), Verständnis und Anwendung der justiziellen Auslegung des Obersten Volksgerichts über die Entschädigung für Personenschä$\operatorname{den}$ (最高人民法院人身损害赔偿司法解释的理解与适用), Beijing 2004, S. 357 f.

40 Das Stadt-Land-Doppelmodell bezieht sich auf die Berechnung des Todes- und Behinderungsentschädigungsgeldes auf der Grundlage der unterschiedlichen Identität von Stadt- oder Landbewohnern und deren verfügbaren Pro-Kopf-Einkommens. In $\$ 29$ der „Auslegung des Obersten Volksgerichts zu einigen Fragen der Rechtsanwendung bei der Behandlung der Entschädigung für Personenschäden“ heißt es: „Das Todesentschädigungsgeld basiert auf dem verfügbaren ProKopf-Einkommen der Stadtbewohner oder das Pro-Kopf-Nettoeinkommen der Landbewohner im Vorjahr, in dem sich das Gericht befindet. Der Betrag wird auf der Grundlage von 20 Jahren berechnet. Für Personen über 60 Jahre wird das Alter jedoch für jedes weitere Jahr um ein Jahr gesenkt; für Personen über 75 Jahre alt wird es als fünf Jahre berechnet. “

41 最高人民法院关于审理人身损害赔偿案件适用法律若干问题的解释 v. 26.12.2003, Amtsblatt des Obersten Volksgerichts (最高人民法院公报) 2004, Nr. 2, S. 3 ff.; deutsche Übersetzung siehe Matthias Göbel (Übersetzer), in: Zeitschrift für Chinesisches Recht 2004, S. 287 ff. 
kommnung des Mechanismus und des politischen Systems zur Entwicklung der Stadt-Land-Integration"42 veröffentlicht, in denen $\$ 17$ eine Reform der Entschädigung für Personenschäden und die Vereinheitlichung der Entschädigungsstandards für Stadt- und Landbewohner deutlich vorschlägt. Im Anschluss daran veröffentlichte das Oberste Volksgericht im September 2019 die „Bekanntmachung über die Ermächtigung für das Pilotprogramm der Vereinheitlichung der Entschädigung für Personenschäden zwischen Stadt und Land“ ${ }^{“ 3}$, in der alle Obervolksgerichte ermächtigt werden, Pilotarbeiten zur Vereinheitlichung der Entschädigung für Personenschäden für Stadt- und Landbewohner nach den spezifischen Bedingungen der jeweiligen Provinz durchzuführen.

Da die spezifischen Umstände jedes Opfers unterschiedlich sind, sollte objektiv gesehen auch die Höhe des Todes- und Behinderungsentschädigungsgeldes von Person zu Person variieren. Daher kann das einheitliche Modell nur die formale Gerechtigkeit erreichen, während die materielle Gerechtigkeit in einem individuellen Modell, das auf individuellen Umständen basiert, zu erwarten ist. Manche Wissenschaftler haben vorgeschlagen, dass bei reifen Bedingungen schrittweise auf ein begrenztes individualisiertes Modell umgestellt werden muss, damit die Höhe der Entschädigung hauptsächlich von den persönlichen Faktoren abhängt, wie Alter, Einkommen, familiäre wirtschaftliche Situation und Entwicklungsperspektiven usw. ${ }^{44}$ Einige Wissenschaftler haben auch aus rechtsökonomischer Sicht geforscht und interne Parameter wie Alter, Kosten, Einkommen, Konsum, Bildung und Risikobereitschaft aufgelistet, die eine entscheidende Rolle bei der Entschädigung spielen sollten. ${ }^{45}$ Wenn der Richter jedoch verpflichtet ist, die oben genannten verschiedenen Faktoren in jedem relevanten Fall umfassend zu berücksichtigen, um den durch die Verletzung oder den Tod einer Person verursachten Einkommensverlust vorherzusagen, kommt dies einer Fantasie gleich.

42 中共中央国务院关于建立健全城乡融合发展体制机制和政策体系的意见 v. 15.4.2019, Amtsblatt des Staatsrates (国务院公报) 2019, Nr. 14, S. 11 ff.

43 最高人民法院关于授权开展人身损害赔偿标准城乡统一试点的通知 v. 2.9.2019.

44 ZHANG Xinbao (张新宝), Erforschung von Todesersatz wegen unerlaubter Handlung (侵权死亡赔偿研究), Chinese Journal of Law (法学研究) 2008, Nr. 4, S. 51 .

45 LI Bensen (李本森), Rechtliche und ökonomische Analyse des Wertes des Lebens - der Reformpfad des chinesischen Gesetzes über die Entschädigung für das Leben (生命价值的法律与经济分析——中国生命赔偿法律的改革路径), Social Sciences in China (中国社会科学) 2011, Nr. 6, S. 88. 
Mit der schrittweisen Anwendung von Big Data und künstlicher Intelligenz in der Justiz hat die Realisierung des individuellen Modells auch einen Hoffnungsschimmer gezeigt. Denn aus Sicht der künstlichen Intelligenz hat jeder einen Code in seinem Körper versteckt. Dieser Code bestimmt nicht nur, was eine Person im realen Zustand tut, sondern repräsentiert auch ihr persönliches Entwicklungspotential und der Algorithmus ist das technische Mittel, das Potential freizuschalten. ${ }^{46}$ Mit anderen Worten, wenn die umfangreichen sozialen und ökonomischen Daten in ein Algorithmus-System eingegeben werden und die Daten sortiert und analysiert werden, kombiniert mit den wichtigen Informationen der Parteien wie dem Bildungsniveau, den zwischenmenschlichen Beziehungen, der Konsumpräferenz, dem Gesundheitszustand, der Risikobereitschaft usw., ergänzt durch normative Überlegungen, dann kann der persönliche Wert relativ genau vorhergesagt werden. Dementsprechend können die durch Behinderung oder Unfalltod verursachten Vermögensschäden berechnet werden.

Wie bereits erwähnt, eignet sich das Gebiet des zivilrechtlichen Schadensersatzrechts möglicherweise besser für die Anwendung von Algorithmen zur Prognose, als es bei Strafverfahren der Fall ist. Der Richter kann bei der Verwendung von Algorithmen zur Vorhersage des zukünftigen Einkommens einer Person in einem Fall das Ergebnis relativ intuitiv mit ähnlichen Fällen vergleichen. Wenn der Algorithmus ein abnormales Ergebnis liefert, kann der Richter es leicht finden und eingreifen, um blindes Vertrauen in den Algorithmus zu vermeiden.

Obwohl der Algorithmus auf Big Data basiert, kann sein vorhersagbarer Bereich nicht alle Situationen abdecken. Beispielsweise sind für Kinder viele Schlüsselindikatoren noch nicht reif, sodass die Genauigkeit der Prognose in solchen Situationen stark abnimmt. Daher kann die algorithmische Prognose für manche Gruppen nur als Referenz verwendet werden, die noch normative Ergänzungen erfordert. Es ist einzuräumen, dass Algorithmen die Zukunft in keiner Weise genau vorhersagen können, sondern nur die Situation mit höchster Wahrscheinlichkeit durch mathematische Modelle kalkulieren. Trotzdem ist die algorithmische Prognose in absehbarer Zukunft der einzig verlässliche Weg, um das individuelle Modell der Entschädigung für Personenschäden zu realisieren. Im Vergleich zu dem Stadt-Land-Doppelmodell und dem einheitlichen Modell entspricht es eher den Anforderungen der materiellen Gerechtigkeit. Obwohl der Gesetzgeber und die Rechtsprechung noch keine Algorithmen zur Berech-

46 John Cheney-Lippold (Fn. 2), S. 262 ff. 
nung und Prognose des Todes- und Behinderungsentschädigungsgeldes verwendet haben, ist es meines Erachtens Zeit, einen mutigen Schritt vorzutreten.

\section{Hindernisse und Herausforderungen der algorithmischen Prognose}

Die Prognosefunktion des Algorithmus weist eine hohe Effizienz auf, die vom Menschen nicht erreicht werden kann. Sie kann die Einschränkungen der Richter wirksam ausgleichen und hat eine sehr breite Entwicklungsperspektive. Vor der Realisierung gibt es jedoch noch einige Hindernisse und Herausforderungen, die nacheinander überwunden werden müssen.

\section{Quelle der Justizdatenbank}

Auf dem Gebiet von Informatik und Big Data gibt es ein allgemeines Sprichwort, „garbage in, garbage out", das bedeutet, dass wenn schlechte Daten in den Computer eingegeben werden, unvermeidlich eine falsche Ausgabe erzeugt wird. Ob die algorithmische Prognose den Tatsachen nahekommt, hängt weitgehend davon ab, ob die zugrundeliegenden Informationen genau, detailliert und reichhaltig genug sind. Für die Anwendung der algorithmischen Prognose in der Justiz ist daher die Bedeutung einer umfassenden Justizdatenbank selbstverständlich.

Im Systemprojekt zum Aufbau einer großen Justizdatenbank in China ist die Online-Veröffentlichung der Entscheidungsdokumente einer der Kernschritte. Das chinesische Oberste Volksgericht hat in den Jahren 2010, 2013 und 2016 dreimal die „Bestimmungen zur Veröffentlichung von Entscheidungen durch Volksgerichte im Internet“47 erlassen, um die OnlineVeröffentlichung der Entscheidungsdokumente zu fördern. Zunächst gab es jedoch keine einheitliche Online-Plattform für die Veröffentlichung, sodass die 2010 erlassenen Bestimmungen keine wesentlichen Auswirkungen hatten. ${ }^{48}$ Am 1. Juli 2013 wurde die Website „China Judgements Online“

47 最高人民法院关于人民法院在互联网公布裁判文书的规定 v. 21.11.2010; 最高人 民法院关于人民法院在互联网公布裁判文书的规定 v. 21.11.2013, Amtsblatt des Obersten Volksgerichts (最高人民法院公报) 2014, Nr. 5, S. 6 ff.; 最高人民法院关 于人民法院在互联网公布裁判文书的规定 v. 29.8.2016, Amtsblatt des Obersten Volksgerichts (最高人民法院公报) 2016, Nr. 11, S. 3 ff.

48 MA Chao (马超)/YU Xiaohong (于晓虹)/HE Haibo (何海波), Big-Data-Analyse: Bericht über online veröffentlichte Entscheidungen in China (大数据分析 : 中国 
eröffnet, um die Lücke in der einheitlichen Veröffentlichungsplattform zu schließen. Wegen der „Bestimmungen zur Veröffentlichung von Entscheidungen durch Volksgerichte im Internet“ 2013 und 2016 stieg die Internet-Zugangsrate von Entscheidungsdokumenten von etwa 50\% in 2014 auf über $70 \%$ in $2018 .{ }^{49}$ Bis Ende August 2020 hat die Gesamtzahl der online veröffentlichten Entscheidungsdokumente 100 Millionen überschritten. Laut Statistiken verschiedener Parteien werden jedoch mehr als $20 \%$ der nach dem Gesetz zu veröffentlichenden Entscheidungsdokumente noch nicht online veröffentlicht. ${ }^{50}$ Daher besteht immer noch eine große Lücke zwischen der tatsächlichen Situation und dem Ziel der vollständigen Offenlegung.

Darüber hinaus gibt es auch Probleme mit der Qualität der bereits veröffentlichten Entscheidungsdokumente. Eine herausragende Situation solcher Probleme liegt darin, dass die Informationen, die der Außenwelt zur Verfügung stehen, nicht unbedingt die „wesentlichen Informationen“ sind, die tatsächlich vom Richter bei der Entscheidungsfindung verwendet werden. ${ }^{51}$ Darüber hinaus werden die Entscheidungen häufig durch den subjektiven Willen und das Werturteil des Richters beeinflusst, was zum sog. „Unterschiedliche-Entscheidungen-für-ähnliche-Fälle“-Problem führt. Wenn solche Entscheidungen ohne Überprüfungen von der Justizdatenbank aufgenommen werden, wird auch die Objektivität der Daten beeinträchtigt. ${ }^{52}$ Ein weiteres Problem, das nicht hätte auftreten dürfen, bestand darin, dass Probleme wie das falsche Zitieren von gesetzlichen Vorschriften auch in manchen Entscheidungsdokumenten auftauchten, was sich

司法裁判文书上网公开报告), China Law Review (中国法律评论) 2016, Nr. 4, S. 242.

49 Vgl. TANG Yingmao (唐应茂), Gerichtliche Offenlegung und ihre Determinanten: Datenanalyse basierend auf Daten von China Judgements Online (司法公开 及其决定因素：基于中国裁判文书网的数据分析), Qinghua University Law Journal (清华法学) 2018, Nr. 4, S. 42; YANG Jinjing (杨金晶)/QIN Hui (覃 慧)/HE Haibo (何海波), Chinesische Praxis über die Online-Veröffentlichung der Entscheidungsdokumente - Fortschritte, Probleme und Verbesserungen (裁判文 书上网公开的中国实践——进展、问题与完善), China Law Review (中国法律 评论) 2019, Nr. 6, S. 129.

50 YANG Jinjing/QIN Hui/HE Haibo (Fn. 49), S. 129.

51 ZUO Weimin (左卫民), Gedanken zu den Perspektiven der Verwendung der legalen künstlichen Intelligenz in China (关于法律人工智能在中国运用前景的若 干思考), Qinghua University Law Journal (清华法学) 2018, Nr. 2, S. 115.

52 ZUO Weimin (Fn. 51), S. 116. 
auch negativ auf die Justizdatenbank auswirkte. ${ }^{53}$ Daher sollten nicht nur immer mehr Entscheidungsdokumente online veröffentlicht werden, sondern auch die Verbesserung der Qualität von Urteilsdokumenten beachtet werden.

Darüber hinaus sollte der Aufbau einer großen Justizdatenbank nicht auf die Online-Veröffentlichung von Entscheidungsdokumenten beschränkt werden. Denn im gesamten Gerichtsverfahren ist das Entscheidungsdokument nur ein wichtiger Teil davon. Andere Teile wie Beweismittel, Gerichtsverhandlungen, Akten und Richterdiskussionen sind auch wertvoll. Glücklicherweise hat das Oberste Volksgericht dies bereits erkannt und im Juli 2015 das Konzept des „intelligenten Gerichts“ vorgeschlagen. ${ }^{54}$ Seitdem haben Gerichte auf allen Ebenen in China fruchtbare Ergebnisse beim Aufbau spezieller Netzwerke, bei der Online-Verarbeitung aller Services, bei elektronischen Rechtsstreitigkeiten, bei der automatischen Spracherkennung bei Gerichtsverhandlungen, bei der Digitalisierung der Akten und beim Aufbau von Justizdatenbanken erzielt. ${ }^{55}$ Diese Fortschritte ermöglichen es, den gesamten Prozess der Justiz zu digitalisieren und eine richtige und umfangreiche Justizdatenbank aufzubauen.

\section{Die über die justiziellen Daten hinausgehende rechtliche Big Data}

Für die justizielle Anwendung der algorithmischen Prognose sind Justizdaten wie Entscheidungsdokumente nur die Spitze des Eisbergs. Wirklich nützliche Daten sollten auch solche Daten enthalten, die sich auf die Gesetzgebung, Verwaltung, Rechtsdienstleistungen sowie andere einschlägige Daten beziehen, nämlich die sog. rechtliche Big Data, die über die justiziellen Daten hinausgeht. Der Aufbau einer solchen Rechtsdatenbank beruht dann auf der Zusammenarbeit und Kooperation verschiedener staatlicher Behörden. Beispielsweise bezieht sich das individuelle Modell der Entschädigung für Personenschäden auf die Behörden der Bevölkerung, Bildung, Polizei, Justiz usw.

53 WANG Zhu (王竹), Die Verbesserung von Qualität und Quantität der rechtlichen Big Data soll berücksichtigt werden (法律大数据要注重质与量的提升), Social Sciences Weekly (社会科学报) 2.6.2016, S. 4 .

54 DENG Heng (邓恒), Wie man intelligentes Gericht und Internetgericht versteht (如何理解智慧法院与互联网法院), People's Court Daily (人民法院报) 25.7.2017, S. 2.

55 Vgl. Chinesisches Oberstes Volksgericht (Hrsg.), Justizreform der chinesischen Gerichte (2013-2018) (中国法院的司法改革<2013-2018>), Beijing 2019, S. 59 ff. 
Es ist jedoch äußerst schwierig, eine so große und umfassende Datenbank aufzubauen, die fast „alle Daten“ enthält. ${ }^{56}$ Darüber hinaus sind die Daten unter Kontrolle verschiedener Behörden in China eigentlich „Dateninseln", die nicht miteinander geteilt werden. Und alle Behörden sind unabhängig in der Datenerfassung, -speicherung und -nutzung und bilden jeweils ein System ohne technischen Anschluss untereinander. ${ }^{57}$ Das einfachste Beispiel ist, dass es in verschiedenen Datensystemen mehrere Namen für dasselbe Objekt gibt. In Bezug auf den Begriff der Landbewohner werden beispielsweise Bezeichnungen wie ländliche Bewohner (农村居 民), dörfliche Bewohner (乡村居民), ländliche Haushalte (农村住户) usw. in verschiedenen offiziellen Dokumenten aufgeführt. Dies ist fast fatal für die Big-Data-Analyse. In diesem Fall ist es noch schwieriger, durch Big-Data-Analyse genaue Ergebnisse zu erhalten, selbst wenn alle Behörden gegenseitig Daten austauschen würden. Daher müssen übergeordnete staatliche Stellen über genügend Mut und Entschlossenheit verfügen, um abteilungsübergreifend zu koordinieren, einheitliche Datenspeicherungsverfahren einzuführen und technische Hindernisse zu beseitigen, um eine effektive Zusammenführung massiver Datenmengen aus verschiedenen Bereichen zu erreichen und die Grundlage der Big-Data-Analyse zu festigen.

\section{Rechtliche Beschränkungen für die Verarbeitung personenbezogener Daten}

Voraussetzung für den Aufbau einer großen Rechtsdatenbank ist die $\mathrm{Zu}$ sammenführung umfangreicher Daten, die von verschiedenen Behörden gesammelt und gespeichert werden, um weitere Analyse und Nutzung zu ermöglichen. Dabei sollte der Schutz personenbezogener Daten besonders berücksichtigt werden.

Am 20.8.2021 wurde das Gesetz zum Schutz personenbezogener Daten der Volksrepublik China ${ }^{58}$ verkündet, das am 1.11.2021 in Kraft tritt. $\$ 6$ Abs. 1 davon sieht den Grundsatz der "Zweckbindung" deutlich vor, dass die Verarbeitung personenbezogener Daten einen klaren und angemesse-

56 WU Jing (吴静), Algorithmus ist König: Die „unsichtbare Hand“ in der Big-DataÄra (算法为王：大数据时代 “看不见的手”), Journal of Huazhong University of Science and Technology (Social Science Edition) (华中科技大学学报 $<$ 社会科学 版>) 2020, Nr. 2, S. 12.

57 CHENG Jinhua (程金华), Die Zukunft ist noch nicht gekommen: Überdenken der Infrastruktur von Chinas legalen Big Data (未来还未来：反思中国法律大数 据的基础建设), China Law Review (中国法律评论) 2018, Nr. 2, S. 165 ff.

中华人民共和国个人信息保护法 v. 20.8.2021. 
nen Zweck haben und in direktem Zusammenhang mit dem Verarbeitungszweck stehen und eine Methode anwenden, die die Persönlichkeitsrechte und -interessen am wenigsten beeinträchtigt. In $\$ 6$ Abs. 2 wird vorgeschrieben, dass die Erhebung personenbezogener Daten auf das für die Verarbeitungszwecke erforderliche Mindestmaß zu beschränken ist und personenbezogene Daten nicht übermäßig erhoben werden dürfen, also der Datenminimierungsgrundsatz. Daneben sehen $\$ 1035$ ZGB $^{59}$ und $₫ 41$ und 42 Cybersicherheits $\mathrm{G}^{60}$ auch vor, dass bei der Sammlung und Verarbeitung personenbezogener Daten die Grundsätze der Rechtmäßigkeit, Fairness und Notwendigkeit zu befolgen sind.

Daher müssen wir beim Aufbau einer großen Rechtsdatenbank in China, insbesondere, wenn gewinnererzielende Einrichtungen teilnehmen, besonders den Schutz personenbezogener Daten beachten. Dies wird zwar Einschränkungen für verschiedene Behörden verursachen, aber da$\mathrm{zu}$ beitragen, die Standardisierung und Legalisierung der Verarbeitung personenbezogener Daten durch öffentliche und private Einrichtungen in China zu fördern, was auch dem allgemeinen Trend zur Stärkung des Schutzes personenbezogener Daten in der Welt entspricht.

\section{Programmierung des Algorithmus und algorithmische Diskriminierung}

Big Data erzeugen selbst keinen Wert. Daher ist es notwendig, durch technische Mittel wie Algorithmen die darin verborgenen Informationen abzubauen. ${ }^{61}$ Derzeit behaupten viele Unternehmen der Rechtstechnologie im Bereich der chinesischen künstlichen Intelligenz, fortschrittliche „Deep-learning-neuronaler-Netzwerke“-Algorithmen zu verwenden. Objektiv gesehen gibt es in China jedoch eine klare Trennung zwischen dem Rechtsbereich und dem Bereich der künstlichen Intelligenz: Den Juristen fehlen die Kenntnisse über künstliche Intelligenz; umgekehrt versteht

59 中华人民共和国民法典 v. 28.5.2020, Amtsblatt des ständigen Ausschusses des Nationalen Volkskongresses (全国人民代表大会常务委员会公报) 2020, Sonderausgabe, S. 1 ff.; deutsche Übersetzung siehe Yijie DING/Peter Leibküchler/Nils Klages/Knut Benjamin Pißler (Übersetzer), in: Zeitschrift für Chinesisches Recht 2020, S. $207 \mathrm{ff}$.

60 中华人民共和国网络安全法 v. 7.11.2016; deutsche Übersetzung siehe Peter Leibküchler (Übersetzer), in: Zeitschrift für Chinesisches Recht 2018, S. $113 \mathrm{ff}$.

61 WANG Lusheng (王禄生), Risiken und ethische Regulierung der justiziellen Big Data und Technologie für künstliche Intelligenz (司法大数据与人工智能技术应 用的风险及伦理规制), Studies in Law and Business (法商研究) 2019, Nr. 2, S. 102. 
technisches Personal für künstliche Intelligenz aufgrund fehlender juristischer Grundkenntnisse häufig nicht die Produktanforderungen, die von Juristen gestellt werden. ${ }^{62}$ Daher hat die künstliche Intelligenz im Recht in der Praxis keine wesentlichen Durchbrüche erzielt. ${ }^{63}$ Die Programmierung des Algorithmus, auf den die Juristen hoffen, ist zu einer großen Herausforderung für die künstliche Intelligenz im Recht geworden.

Darüber hinaus erhält der Algorithmus, wie der oben genannte Fall „three black teenagers“ zeigt, hauptsächlich Daten aus dem Internet, kann sich jedoch hinsichtlich der Ergebnisse der Berechnung nicht überprüfen. Selbst wenn der Programmierer des Algorithmus subjektiv keine diskriminierende Absicht hat und auch objektive und neutrale Basisdaten verwendet, kann es zu diskriminierenden Konsequenzen führen. ${ }^{64}$ Es ist einzuräumen, dass es noch viele diskriminierende Phänomene in der Gesellschaft insbesondere im Internet gibt, die beispielsweise Geschlechterdiskriminierung, regionale Diskriminierung, berufliche Diskriminierung usw. umfassen. Wenn der Algorithmus diese Informationen wahllos erhält, ist es auch wahrscheinlich, dass er eine diskriminierende Verzerrung erzeugt und diesen Eindruck im Prozess des Weiteren Erhaltens von Informationen weiter verstärkt, was dazu führt, dass die Ergebnisse der Berechnung beeinflusst werden. Insbesondere bei der Anwendung algorithmischer Prognosen im Bereich der Entschädigung für Personenschäden ist es unvermeidlich, sensible Parameter wie Geschlecht, Region, ethnische Zugehörigkeit u. a. einzugeben. ${ }^{65}$ Aus diesem Grund kann es noch wahrscheinlicher sein, diskriminierende Ergebnisse zu erhalten. Daher ist es notwendig, durch manuelle inhaltliche Überprüfung die Lücke der algorithmischen Selbstprüfung zu schließen und zu verhindern, dass diskriminierende Faktoren in Entscheidungen einfließen. Wenn man das Problem vollständig lösen will, muss die gesamte gesellschaftliche Atmosphäre verbessert werden.

62 ZUO Weimin (Fn. 51), S. $118 \mathrm{ff}$.

63 Vgl. ZUO Weimin (左卫民), Heiß und kalt: Überdenken der legalen künstlichen Intelligenz in China (热与冷：中国法律人工智能的再思考), Global Law Review (环球法律评论) 2019, Nr. 2, S. 55-56.

64 ZHENG Zhihang (郑智航)/XU Zhaoxi (徐昭曦), Rechtliche Regulierung und justizielle Überprüfung der Algorithmusdiskriminierung in der Big Data Ära am Beispiel der amerikanischen Rechtspraxis (大数据时代算法歧视的法律规制 与司法审查——以美国法律实践为例), Journal of Comparative Law (比较法研 究) 2019, Nr. 4, S. 112.

65 ZHANG Endian (张恩典), Schutz vor algorithmischer Diskriminierung: Theoretische Reflexion und institutioneller Aufbau (反算法歧视：理论反思与制度建 构), Journal of Huazhong University of Science and Technology (Social Science Edition) (华中科技大学学报 $<$ 社会科学版 $>$ ) 2020, Nr. 5, S. 61 . 
Wenn es in der Gesellschaft immer weniger Diskriminierungen gibt, wird der Algorithmus auch weniger diskriminierende Informationen erhalten und das Ergebnis wird gerechter.

\section{Fazit}

Im Zeitalter der künstlichen Intelligenz werden Big Data und Algorithmen zunehmend in der Justiz eingesetzt. Während die Arbeitseffizienz verbessert und die Justiz entlastet wird, muss man auch wachsam bleiben, dass die Entscheidung des Richters allmählich durch die algorithmische Entscheidung ersetzt wird. Obwohl die algorithmische Entscheidung in manchen Aspekten Vorteile gegenüber der Entscheidung des Richters hat, weist sie auch schwerwiegende Mängel auf, da sie die Anforderungen der Gerichtsverfahren nicht vollständig erfüllt. Vor dem Hintergrund der künstlichen Intelligenz im Recht ist es daher unerlässlich, darauf zu bestehen, dass menschliche Richter für die Justizhoheit zuständig sind.

Verglichen mit den Risiken der algorithmischen Entscheidung stellt es eine praktikable Möglichkeit dar, die algorithmische Prognosefunktion in der Justiz einzusetzen. Beispielsweise im Bereich der Entschädigung für Personenschäden entspricht es eher den Anforderungen der materiellen Gerechtigkeit, wenn der Algorithmus Prognosen macht, die Menschen nicht leicht erreichen können. Um das Ziel zu verwirklichen, ist eine umfangreiche Rechtsdatenbank sowohl bezüglich „Quantität“ als auch „Qualität" aufzubauen und sind ideale Algorithmen zu programmieren. Gleichzeitig ist der Schutz personenbezogener Daten und der Schutz vor algorithmischer Diskriminierung zu beachten. 